\title{
Tumor and reproductive traits are linked by RNA metabolism genes in the mouse ovary: a transcriptome-phenotype association analysis
}

\author{
Ulises Urzúa 1*, Garrison A Owens², Gen-Mu Zhang ${ }^{3}$, James M Cherry², John J Sharp ${ }^{4}$, David J Munroe² \\ From 5th International Conference of the Brazilian Association for Bioinformatics and Computational Biology \\ (X-meeting 2009) \\ Angra Dos Reis, RJ, Brazil. 18-22 October 2009
}

\begin{abstract}
Background: The link between reproductive life history and incidence of ovarian tumors is well known. Periods of reduced ovulations may confer protection against ovarian cancer. Using phenotypic data available for mouse, a possible association between the ovarian transcriptome, reproductive records and spontaneous ovarian tumor rates was investigated in four mouse inbred strains. NIA15k-DNA microarrays were employed to obtain expression profiles of BalbC, C57BL6, FVB and SWR adult ovaries.

Results: Linear regression analysis with multiple-test control (adjusted $p \leq 0.05$ ) resulted in ovarian tumor frequency (OTF) and number of litters (NL) as the top-correlated among five tested phenotypes. Moreover, nearly one-hundred genes were coincident between these two traits and were decomposed in $76 \mathrm{OTF}(-) \mathrm{NL}(+)$ and 20 $\mathrm{OTF}(+) \mathrm{NL}(-)$ genes, where the plus/minus signs indicate the direction of correlation. Enriched functional categories were RNA-binding/mRNA-processing and protein folding in the OTF(-) NL(+) and the OTF(+) NL(-) subsets, respectively. In contrast, no associations were detected between OTF and litter size (LS), the latter a measure of ovulation events in a single estrous cycle.

Conclusion: Literature text-mining pointed to post-transcriptional control of ovarian processes including oocyte maturation, folliculogenesis and angiogenesis as possible causal relationships of observed tumor and reproductive phenotypes. We speculate that repetitive cycling instead of repetitive ovulations represent the actual link between ovarian tumorigenesis and reproductive records.
\end{abstract}

\section{Background}

Epidemiological evidence indicates that multiparity and breastfeeding as well as endocrine disrupting agents used in oral contraception, hormone replacement therapy and infertility treatment- modulate the risk of ovarian cancer [1]. Repetitive lifetime ovulations would induce a persistent wound repair process of the ovarian surface epithelium cells leading to pre-neoplastic alterations [2]. In addition, oral contraceptives and pregnancy reduce levels of circulating gonadotropins whereas

\footnotetext{
* Correspondence: uurzua@med.uchile.cl

'Laboratorio de Genómica Aplicada, ICBM, Universidad de Chile, Independencia 1027, Santiago, Chile Full list of author information is available at the end of the article
}

fertility drugs induce follicle-stimulating hormone (FSH) production. Gonadotropins also increase with reproductive ageing, and have been implicated in ovarian cancer etiology since this malignancy predominantly occurs in menopausal women [3].

The laboratory mouse has been increasingly used to model several aspects of ovarian cancer [4]. Indeed, reproductive biology of mouse resembles human reproduction in many aspects. Analogous to menstrual cycles in women, female mice undergo estrous cycles that last 4-5 days and consist of four successive phases. Proestrus and estrous phases together constitute the follicular phase while metestrus and diestrus phases together represent the luteal phase [5]. Similar to humans, the
C Biomed Central 
length of estrous cycles increases while the monthly cycle frequency decreases in ageing mice [6]. The number of ovulations roughly reaches 500 during the reproductive life of women while in mice this number can be achieved earlier than middle age due to multiple ovulations in a single cycle [7] as judged by the litter size observed in mice [5]. Cysts, invaginations and cell layering are also common observations in the mouse and human ovaries [7].

Mouse inbred strains display measurable traits that are described as continuous phenotypes in the Mouse Phenome [8] and the Mouse Tumor Biology [9] databases. The natural variability observed in mice strains offers the opportunity to study disease susceptibility in a genetically defined background. In simple terms, phenotypic variability could be due to the interplay of gene transcripts and/or proteins expressed at different relative abundances across individuals in a tissue or cell type implicated in a phenotype. Thus, if a correlation exists between a continuous phenotype and gene expression, a measure of each gene's contribution to the observed phenotype can be inferred. DNA microarrays are suited to measure transcript levels for hundreds or thousands of genes simultaneously, and thus such contribution can be addressed in a wide-genome format. A number of statistical approaches have been recently formulated to correlate DNA microarray data with phenotypic covariates [10].

In an attempt to gain novel information linking reproductive parameters with ovarian tumorigenesis we describe here a correlation analysis between spontaneous ovarian tumors, reproductive phenotypes and gene expression profiles obtained with NIA15k-DNA microarrays from ovaries of four mouse inbred strains. Using a linear regression approach with control of multiple testing, "ovarian tumor frequency" (OTF) and "number of litters" (NL) were the top-correlated of 5 analyzed phenotypes. About one hundred genes were coincident between OTF and NL. The enriched biological functions in this overlapped sub-set were "RNAbinding/mRNA-processing" and "protein folding". The relevant information concerning the significant genes was mined and the relationship between ovarian function and ovarian tumorigenesis at the molecular level is discussed.

\section{Results}

\section{Consistency of microarray data and Q-PCR assays}

The robustness and reliability of the mouse NIA-15K cDNA microarray platform has been demonstrated in our previous work $[11,12]$ and by others [13-15]. Experimental design employed a common reference RNA and replicate dye-swap. Raw data was subjected to print-tip loess normalization, a numerical correction based on local deviations across the microarray surface aimed to counteract the inherent noise of these devices [16]. Further adjustment consisted of inter-slide scale normalization, after which statistical comparisons were performed. The complete normalized dataset for 14,586 cDNA clones in 23 microarrays corresponding to 4 samples (i.e. 6 replicates for 3 samples and 5 replicates for 1 sample) is available as a Supplementary spreadsheet (Urzua_complete_dataset.xls) and has been deposited to the GEO database (http://www.ncbi.nlm.nih.gov/geo/) with the accession code GSE18045. One of the 24 hybridization experiments was discarded because not meeting with minimal image quality parameters. Figure 1, panels A through $C$, shows microarray data comparison for expression of genes Tsc22, Col3a1 and Fubp 1 in 2 strains assayed each in 6 microarray replicates. These genes were selected because they were present as multiple cDNA clones in the NIA-15K collection, so that intra-slide consistency could also be evaluated. The expression change (ec), defined as the difference between $\log _{2}$ ratio averages of all clones in the $6 \times 2$ replicate arrays, was similar among the 3 genes. However, the adjusted $p$ value was significant for the Fubp1 gene only. Additionally, quantitative-PCR (Q-PCR) confirmation assays were performed for seven genes assessed in a previous mouse ovarian study, and for which primer pairs were available. Three of these genes showed statistical significance after an ANOVA test across the 4 mouse strains (see next section). The $C_{T}$ values for test and reference samples were corrected with the 18S-rRNA as internal control transcript and then converted to $\log _{2}$-based ratios to compare with microarray results. As shown in Figure 1D, the squared correlation between Q-PCR and microarray platforms was $R^{2}=0.749$, a value over the range observed in a recent large-scale study aimed to validate microarray data using Q-PCR [17]. Microarray data roughly ranged from -4.0 to +1.5 while the correspondent Q-PCR results ranged from -4.9 to +3.4 . Except for one data pair, out of 28 comparisons, the ratio direction (up- or down-regulation) more than the absolute value, was consistent between both methodologies. Additional file 1, Table 1 (Urzua_Suppl_Results) shows detailed microarray $\log _{2}$ ratio values and $C_{T}$ values for the seven genes in all test and reference samples.

\section{Tumor and reproductive parameters correlated with ovarian gene expression}

A preliminary ANOVA analysis resulted in $628 \mathrm{cDNA}$ clones $(4.27 \%$ of the whole dataset) with statistically significant differences (adjusted $p<0.05$ ) between 2 of any of the four mouse ovarian tissue profiles (data not shown). These results simply indicate that transcriptional differences indeed occur among ovarian tissue 

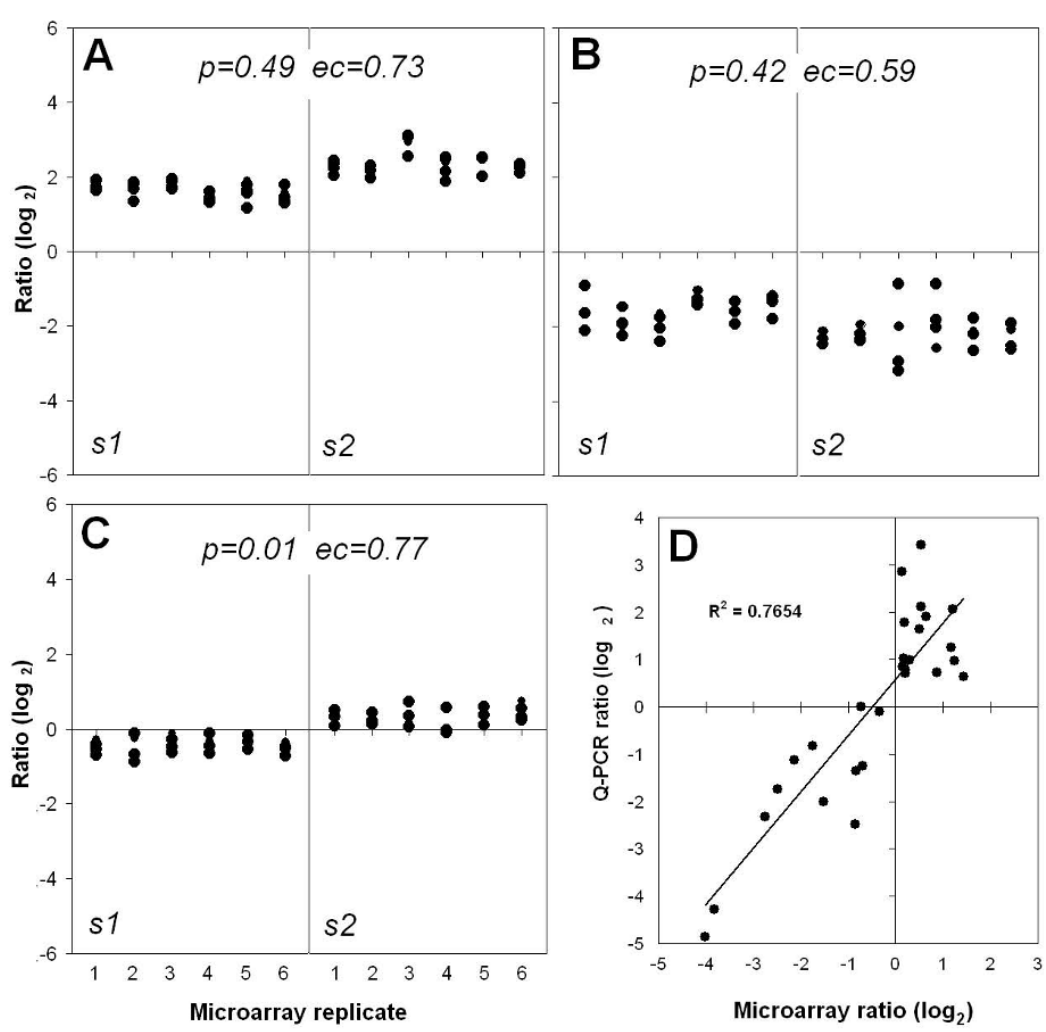

Figure 1 Consistency of microarray results and Q-PCR confirmation of selected genes Normalized microarray data of 5 CDNA clones of Tsc22 (A), 4 clones of Col3al (B) and 4 clones of Fubp1 (C) were compared between samples s1 and s2 (corresponding to 2 of the 4 mouse ovarian samples assayed) across 6 microarray replicates each. Adjusted $p$-values and expression change (ec), defined as the difference between $\log _{2}$ averages are shown. In D, the coordinates of scatter plot depicts the average of normalized microarray ratios (5-6 replicates) and the average of duplicate Q-PCR assays for the genes Spp1, Txnrd1, Anxa5, Fn1, C15, Ctsl, and Mt1 in each of the 4 mouse strains. The internal control transcript was the $18 \mathrm{~S}$ rRNA. Q-PCR data was converted to $\log _{2}$ scale ratios as described in Methods. The squared correlation coefficient $\left(R^{2}\right)$ shown corresponds to the overall gene subset in the 4 strains (i.e. 28 data pairs). Raw Q-PCR results and individual microarray ratios are detailed in Additional file1, table 1.

across strains. Thus, in order to add physiological meaning to this observation, the whole microarray dataset was subjected to a linear regression analysis with each of the continuous covariates shown in Table 1. A summary of regression results is shown in Table 2. Using an adjusted $p$ value $<0.05$ as cut-off, a total of 590 clones that represented 401 known genes, 93 unknown genes, 45 transcribed locus and 9 expressed sequences were significantly correlated to 4 of the 5 traits analyzed. The overlap between the regression analysis and the ANOVA test was 386 clones. The phenotype productive matings (PM) did not result in any significantly

Table 1 Ovarian tumor and reproductive phenotypes in selected mouse strains

\begin{tabular}{lcccc}
\hline Strain & Tumor frequency $^{\mathbf{a}}$ & Litter size $^{\mathbf{b}}$ & Number of litters & Productive matings (\%) $^{\text {Relative fecundity }}$ \\
\hline BALB/C & 3.80 & 4.9 & 3.6 & 55.6 \\
C57BL/6 & 1.60 & 6.6 & 3.8 & 87.4 \\
FVB $^{c}$ & 7.00 & 9.5 & 4.8 & 90.0 \\
SWR & 57.0 & 7.5 & 2.3 & 58.3 \\
\hline
\end{tabular}

" Refers to spontaneously arisen tumors in inbred mice. Data corresponds to the "highest reported tumor frequency" in all literature records collected in the Mouse Tumor Biology Database [9], (http://tumor.informatics.jax.org/mtbwi/dynamicGrid.do;jessession=89370725979E9B939D3DD40AB4961BA5) for each strain/ organ combination where organ=ovary.

b Litter size, number of litters, and productive matings were taken from the Mouse Phenome Database [8] (http://www.jax.org/phenome). Data acquisition, curation and handling are described at http://phenome.jax.org/db/q?rtn=docs/aboutmpd. The parameter "relative fecundity" is derived form the other 3 reproductive parameters [5].

' Data of FVB strain was taken from Silver's Mouse Genetics textbook [5]. 
Table 2 Summary of correlation results between ovarian gene expression and phenotypes

\begin{tabular}{|c|c|c|c|c|c|c|c|c|c|}
\hline \multirow[t]{2}{*}{ Trait } & \multirow[t]{2}{*}{ 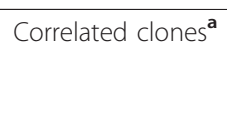 } & \multicolumn{4}{|c|}{ Trait interactions $^{\mathbf{b}}$} & \multicolumn{2}{|c|}{ Direction and strength of correlation ( $R$ value range) ${ }^{c}$} & \multicolumn{2}{|c|}{$\begin{array}{l}\text { Gene expression shift } \\
\left(\delta \log _{2} \text { value range }\right)^{\mathbf{d}}\end{array}$} \\
\hline & & $\overline{\mathrm{OTF}}$ & $\mathrm{NL}$ & LS & $\mathrm{RF}$ & Positive & Negative & Positive & Negative \\
\hline Ovarian tumor frequency (OTF) & $425(280)$ & - & 145 & 0 & 0 & $97(1.00-0.51)$ & $328(1.00-0.52)$ & $3.26-0.19$ & $2.96-0.21$ \\
\hline Number of litters (NL) & $234(82)$ & 145 & - & 0 & 7 & $161(1.00-0.73)$ & $72(0.99-0.67)$ & $2.94-0.32$ & $3.00-0.24$ \\
\hline Litter size (LS) & $73(66)$ & 0 & 0 & - & 7 & $53(0.99-0.78)$ & $20(0.99-0.62)$ & $2.50-0.32$ & $2.18-0.41$ \\
\hline Relative fecundity (RF) & $17(3)$ & 0 & 7 & 7 & - & $12(0.98-0.78)$ & $5(0.98-0.90)$ & $1.50-0.29$ & $0.85-0.51$ \\
\hline
\end{tabular}

${ }^{a}$ Number of clones resulting from a regression analysis with false discovery rate (FDR) control performed with the multiple test tool Pomelo (accessible at http:// pomelo2.bioinfo.cnio.es/). For each trait, genes were filtered with an adjusted $p<0.05$ resulting in a total of 590 unique arrayed clones showing statistically significant correlation. Under this criterion, the trait "productive matings" did not show correlation. The number of clones exclusively correlated with the indicated trait is shown between parentheses.

${ }^{\mathrm{b}}$ Interactions correspond to the number of clones correlated with two or more traits as obtained with Boolean comparisons.

c Pearson correlation coefficients (R) were calculated using gene expression $\log _{2}$ ratios as independent variable and each of phenotypic trait (see Table 1 ) as dependent variables. The observed range of $\mathrm{R}$ values for all correlated genes is shown between parentheses.

${ }^{d}$ Gene expression ratios $\left(\log _{2}\right)$ for the extreme trait values were subtracted $(\delta)$ and then ranked. The highest and the lowest ratio difference are shown between parentheses.

correlated gene. The gene ontology (GO) profile of the 401 known genes included "regulation of transcription" (67 genes; $p=4.4 \mathrm{e}-7$ ), "RNA binding" ( 44 genes; $p=$ 4.3e-16) and "RNA metabolism" (26 genes; $p=1.0 \mathrm{e}-7$ ) with the highest statistical significance in a hypergeometric test. Notably, even though GO terms are inherently redundant, the combined 3 functional groups corresponded to $26 \%$ (105) of known significant genes. The overall functional profile of the 401 genes list is detailed in Additional file 1, table 3. Ovarian tumor frequency (OTF) was the top correlated trait followed by number of litters (NL). The relationship between the squared correlation coefficient $\left(R^{2}\right)$ for each of the 425 OTF correlated clones and its corresponding geneexpression shift is depicted in Figure 2. Negative and positive correlation was observed for 328 and 97 clones, respectively. Notably, a large fraction of strongly correlated genes $\left(R^{2}=0.8\right.$ to 1.0$)$ showed gene-expression shifts under 1 unit in the $\log _{2}$ ratio scale (mean +0.53 and -0.58 in the yellow highlighted quadrants) which equals to less than 2-fold up- or down-regulation in the linear scale across the 4 strains. Selected clones either with high significance, large expression shift or both are tagged with red and green dots in Figure 2. OTF positively correlated genes included the unknown function H3055D10 clone, the BC003993 clone coding for the KIAA1604 protein presumably implicated in nuclear mRNA splicing [18], the heat shock proteins Hspb1, Hsp90aa1 and Dnajb1 involved in protein folding and cellular stress [19], and the gene Star (steroidogenic acute regulatory protein), which mediates mitochondrial cholesterol transport for its conversion to pregnenolone [20]. On the other hand, genes negatively correlated with OTF included Ogt, coding for a $\mathrm{N}$-acetylglucosamine transferase enzyme activity implicated in heat-stress response [21], the mRNA splicing genes Hnrnpa2b1, a possible early detection marker of lung cancer [22], and Sfrs 5 which is overexpressed in breast tumors [23]. Additional OTF(-) genes included 2310043N10Rik corresponding to a virus-inducible non-coding RNA (VINC) expressed in brain and several adult non-neuronal mouse tissues [24], the two clones of Malat1, a long, non-coding metastasis-associated lung cancer transcript up-regulated various tumors including ovarian cancer [25], and Clk1 (CDClike kinase 1), involved in nuclear phosphorylation of serine/arginine-rich proteins in the spliceosomal complex [26].

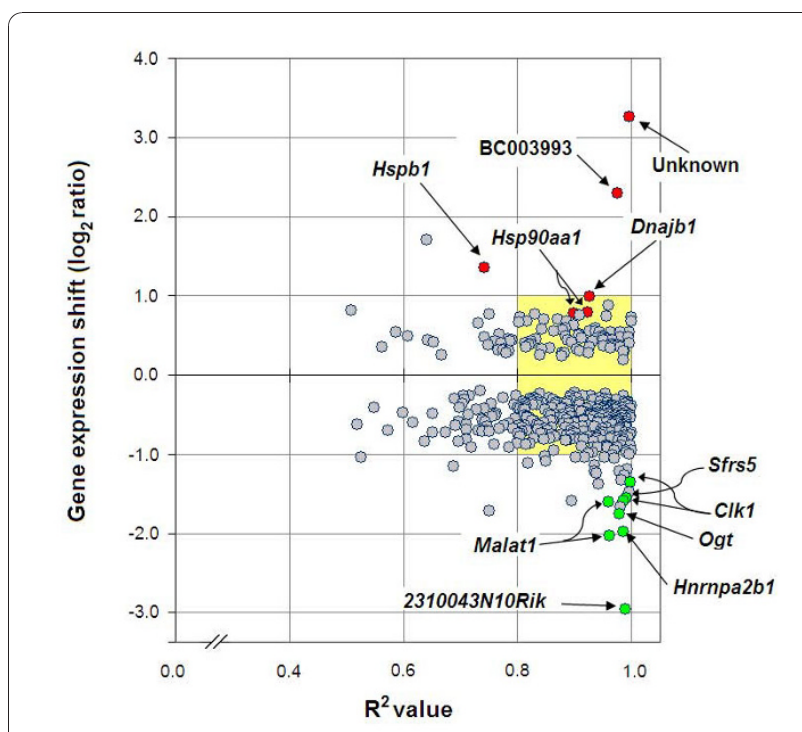

Figure 2 Strength of OTF correlation as a function of ovarian gene expression shift The squared correlation coefficient $\left(R^{2}\right)$ for the 425 clones positively and negatively correlated (adjusted $p<0.05)$ with ovarian tumor frequency (OTF) were plotted against their respective gene expression shifts. The shift is defined as the resultant of the subtracted gene expression $\log _{2}$ ratios between extreme values (see Table 1 and Additional file 1, 2, 3 Results file). 


\section{Functional analysis of genes associated both to} OTF and NL

Table 2 also shows the number of genes correlated with more than one trait. The highest overlap was 145 clones associated both with OTF and NL. No overlap was observed between OTF and litter size (LS), or between
OTF and relative fecundity (RF). The latter parameter -numerically derived from NL, LS and PM [5] - resulted in 17 correlated genes, 14 of them also correlated to NL and LS, 7 genes each trait. Interestingly, LS showed the highest percentage (90\%) of exclusively correlated genes. Figure 3 shows scatter plots for a combined total of 40

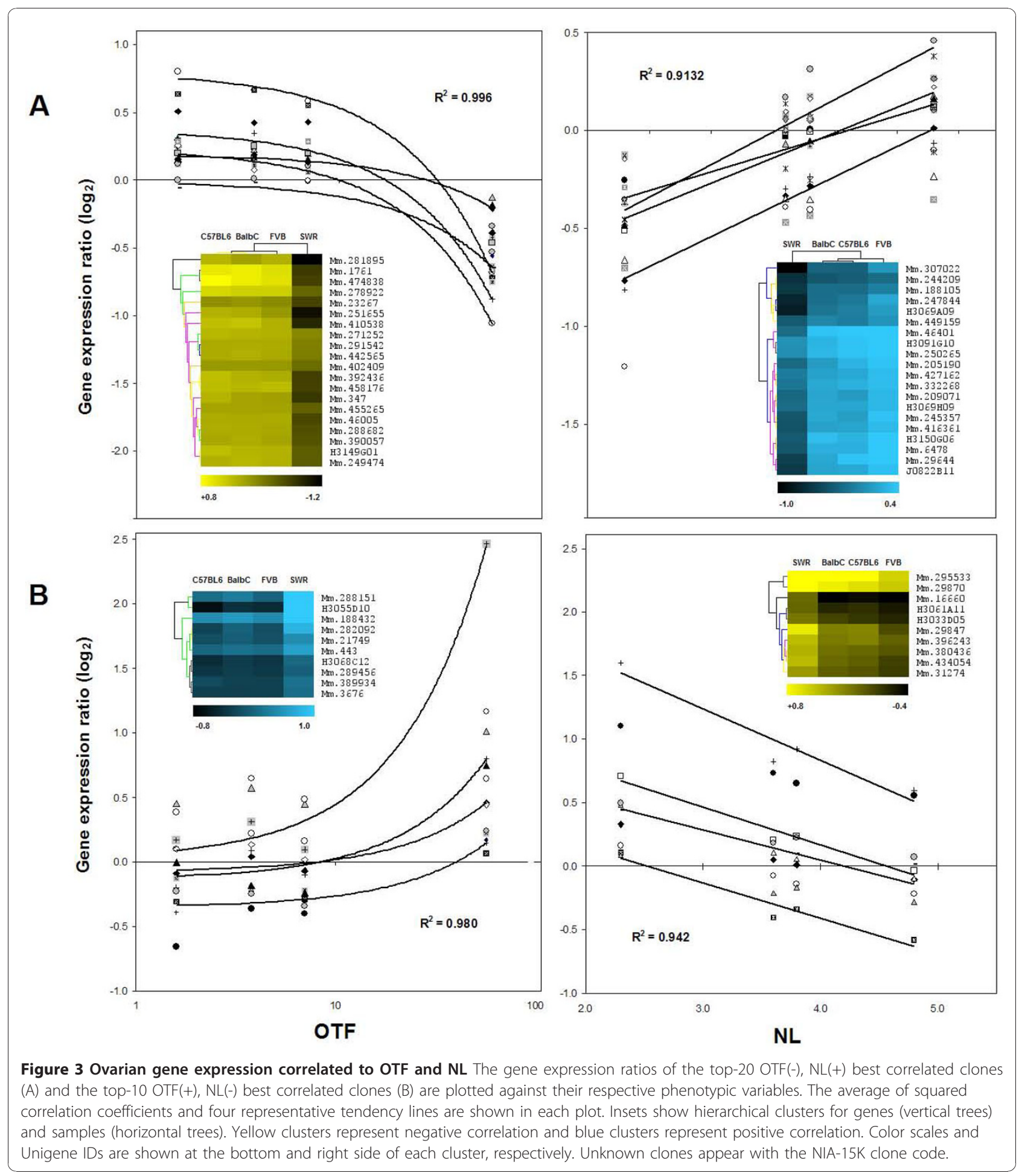


OTF and 20 NL correlated transcripts. Genes with the top correlation coefficients (R) and equivalent distribution of positive and negative correlation are shown. $\mathrm{R}$ values of negatively correlated clones ranged -0.999 to -0.798 , and +0.998 to +0.875 for the positively correlated ones. Similarly, R values for NL correlated genes ranged -0.986 to -0.845 and +0.999 to +0.756 [Additional file 1 , 2, 3 “OTF\&NL_correlated_transcripts.xls"]. Following exclusion of unknown clones and merging of repeats, the OTF/NL-145 clones list was reduced to 117 single Unigene IDs including 21 transcribed loci of known chromosomal location but unassigned biological function. Thus, the final list actually consisted of 96 unique gene identities and was further decomposed in 2 sublists based on direction of correlation: 76 OTF(-) NL(+) genes and $20 \mathrm{OTF}(+) \mathrm{NL}(-)$ genes, where the minus sign indicates negative correlation and the plus sign indicates positive correlation. The genes in enriched functional categories (56 out of the 96) are shown in Additional file 4. Overall, the predominant functions were related to RNA-metabolism (i. e. RNA binding, mRNA processing, regulation of transcription, zinc ion binding) and protein folding and degradation. Eleven of the 56 genes have not been linked to a defined cellular or physiological process since they comprise recently identified genes for which GO terms were inferred from electronic annotation. Importantly, 15 genes are linked to normal or pathological ovarian processes, including Cited 1 and Ece1 directly implicated in reproductive functions. Further links to reproduction involve the genes Aplp2, Dnaja1, Htt, Rps6kb1 and Spin1 which were part of the overall correlated 590 clones subset (see Discussion). In addition, as shown in Additional file 4 , five genes have been described in normal or altered hematopoiesis, 5 genes are associated to neurological disorders, 3 genes are muscle-related and 3 genes are linked to non-ovarian endocrine function.

\section{Discussion}

Complex phenotypes are the outcome of many genes interacting with each other and with endogenous or exogenous factors. Mouse strains displaying phenotype variability allow interrogation on their molecular basis in a particular tissue or condition. In this report, the ovarian expression of roughly 400 genes (corresponding to 590 transcripts in Table 2) was significantly correlated to 4 of 5 mouse tumor and reproductive phenotypes assessed with a linear regression model. The predominant gene ontology (GO) terms were "regulation of transcription", "RNA binding" and "RNA metabolism" accounting for 105 of all correlated genes. A minor, but significant group was "ubiquitin cycle" with 14 genes. Links to reproductive processes are described for Aplp2, Chuk, Dnaja1, Htt, Pten, Rps6kb1, Sf1, Spin1 and Tnc in the GO directory. Rps6kb1 is involved in proliferation of granulosa cells in response to FSH [27]. Rps6kb1 and Chuk, in addition to Nfkb1, Map3k10, Flna, Kras, Rap1a, and Hspb1 belong to the MAP kinase signaling pathway which has been implicated in mammalian oocyte maturation and fertilization [28]. The correlation of Kras (K-Ras 2), commonly mutated in various human tumors, with litter size (LS) can be supported by its involvement in granulosa cell differentiation and ovulation [29].

In a study on null Foxo3 mice, a mutant displaying early ovarian hyperplasia due to synchronous primordial follicle activation, 6 genes (Spin1, Slc45a3, Rspo2, Star, Trim71 and Gm196) present in our 400-genes list were postulated as fertility factors [30]. Star (steroidogenic acute regulatory) protein was positively correlated to OTF (see Figure 2). Star transports cholesterol into the mitochodria, a key process in steroid-hormone synthesis in all major steroidogenic tissues [20]. Additional genes related to steroid metabolism and present in the 400-list included $\mathrm{Hmgcr}$ (3-hydroxy-3-methylglutaryl-CoA reductase), the major regulatory step in cholesterol synthesis; Idi1 (isopentenyl-diphosphate delta isomerase) involved in conversion of mevalonate into activated isoprene units, and Lss (lanosterol synthase) that catalyzes the cyclization of squalene-2,3-epoxide to lanosterol. A recent work has implicated metabolic products of lanosterol in primordial folliculogenesis by regulation of oocyte meiosis and apoptosis [31]. Other indirectly steroid-related gene was Mbtps2, a membrane-embedded zinc metalloprotease which activates signaling proteins involved in transcription induced by steroids [32].

A large portion of the 590 list (24.6\%, i.e. 145 clones) was found to be associated both to spontaneous ovarian tumor frequency (OTF) and number of litters (NL) as shown in Table 2. A link between OTF and NL agrees with an increased risk of ovarian tumorigenesis due to successive menstrual cycles in women. In contrast, conditions that interrupt cycles block ovulations and thus reduce risk [2]. Accordingly, a mouse strain displaying high NL has been subjected to a longer period without cycling than compared with a low NL strain. Successive pregnancies and lactation may be responsible of this effect. We detected a set of 76 mouse genes that were positively correlated to NL, i.e. elevated expression levels were observed in strains showing high NL. Thus, since a concomitant negative correlation was observed with ovarian tumor frequency (OTF), over-expression of these 76 genes set could be considered "protective". By analogy, the 20 genes that were negatively correlated to NL, i.e. down-regulated in high NL mice, may have a role as "susceptibility" genes since they showed a parallel positive correlation with OTF. Importantly, high litter sizes (LS) involve multiple simultaneous ovulations but 
no association was detected between OTF and LS in our data. It may be hypothesized that the damage caused to the epithelial surface during a multiple-ovulation event can be repaired during subsequent pregnancy and lactation, a period without cycling and ovulations.

Additional file 4 shows that 11 of the 76 OTF-/NL+ genes are implicated either in normal or pathological ovarian processes. RNA binding was the predominant GO term in the 76-genes list reaching 22 genes. RNA binding proteins have been implicated in mammalian germ cell development [33]. The genes Cpsf6, $D d x 17$, Fubp1, Hnrpa2b1, Rbm25, Rbm39, Sfrs2 and Sfrs6 share GO terms (RNA binding, mRNA processing) and a relationship with normal ovarian function or ovarian-related disease. Some genes not related to RNA metabolism but linked to ovarian function were correlated to OTF-/NL + . These include Ece1, expressed in steroidogenic and follicular endothelial ovarian cells in a parallel fashion to corpus luteum maturation [34], and the genes Arnt and Nfat5 that regulate the activity of vascular endothelial growth factor (VEGF), an important angiogenesis modulator in normal and pathological conditions including ovarian malignancies [35]. Arnt (alias HIF-beta) binds the hypoxia inducible factor-1 alpha (HIF-1alpha) to form a heterodimer that recognizes the VEGF promoter [36]. Arnt can alternatively bind the aryl hydrocarbon receptor (AHR) forming an AHR/ARNT complex which controls FSH and $\mathrm{LH}$ concentrations in response to AHR ligands [37]. Furthermore, down-regulation of Nfat5 (nuclear factor of activated T-cells 5) parallels a decrease in VEGF's receptor VEGFR1 and an increase in VEGFR2 in hemangioma endothelial cells [38].

Additional regulators of VEGF's function in the OTF-/NL+ list were the genes Sfrs6 and Rbm39. The splicing factor Sfrs6 (alias SRp55) upregulates the antiangiogenic VEGF isoform, an alternative splicing product involving the $8^{\text {th }}$ exon of VEGF's pre-mRNA [39]. Since Sfrs6 displayed an OTF-/NL+ correlation pattern, this is suggestive of an anti-angiogenic condition associated to multiparity and low ovarian tumor incidence. VEGF levels itself were neither differentially expressed nor correlated to any phenotype in this study, but the CDC-like kinase 1 (Clk1), which mediates Sfrs6 activity on anti-angiogenic VEGF levels [39], was also part of the OTF-/NL+ list (see Additional file 3 "OTF\&NL_correlated_transcripts.xls"). In addition, Rbm39 (alias CAPERalpha) is able to alter the VEGF-121/VEGF-189 ratio in breast cancer cells [40]. Rbm39 was originally described as an ER $\alpha / \beta$ transcriptional coactivator [41]. Analogous role has been reported for the DEAD-box RNA helicases $D d x 17$ (alias p72) and $D d x 5$ (alias p68). [42]. $D d x 17$ was in a OTF-/NL+ fashion while $D d x 5$ plus $D d x 26 b, D d x 3 x$ and $D d x 42$ were present in the overall 400-genes list.
Regarding the $\mathrm{OTF}+/ \mathrm{NL}-$ correlated genes, the predominant GO term was "protein folding" including Dnajb1, Hsp90aa1 and P4hb (see Additional file 4 and Figure 2). Dnajb1 is a member of the Hsp40 co-chaperone protein family of which its Drosophyla homolog participates in oogenesis [43]. Related Hsp-40 genes Dnaja1, Dnajb6, and Dnajc7 were present in the overall 400-genes list. Hsp90aa1 expression is up-regulated in ovarian endometriosis [44] while $P 4 h b$ is involved in post-translational modifications of procollagen synthesis [45]. Other transcripts with $\mathrm{OTF}+/ \mathrm{NL}-$ correlation were Spin1 (Spindlin 1), a protein that associates to CPEB, a RNA-binding protein implicated in polyadenylation during meiotic progression in oocytes [46], and the transcription factor Cited1, reported as a FSH regulated gene in human granulosa cells [47].

All the five phenotypes studied are complex and many causal effects are certainly involved. It is quite possible that a large fraction of correlated transcripts may be simply bystanders but not lie behind the measured phenotype. Of the 56 genes listed in Additional file 4, seventeen are classified under the GO term "regulation of transcription". These may be considered "master genes", i.e. encoding for protein products that somehow interact with DNA regulatory sequences or transcriptional multiprotein complexes thus modulating the transcriptional activity of downstream genes. Among the $\mathrm{OTF}-/ \mathrm{NL}+$ correlated genes with roles in regulation of transcription, we identified Fubp1, Rbm39 and Arnt which are directly linked to ovarian biology or disease (see Additional file 4). Fubp1 encodes a ssDNA binding protein that activates the "far upstream element" of cmyc thus stimulating its transcription. Interestingly, promoter regions of the OTF-/NL+ genes Ccnl1, Clk4, Coq10a, Ddx17, Ict1, Zc3h11a and Zc3h7a were found to contain binding sites for Arnt (data not shown). In addition, the genes Hnrpdl, Sltm, Tardbp, Ccnl1, Ccnl2, Dmtf1, Mll3, Mycbp2, Nfat5, Suv420h2, and 1810007 M14 display diverse roles in cancer and developmental processes (see Additional file 4 for References). Special mention deserve the c-myc binding protein Mycbp2, the gene Sltm, which has been described as modulator of estrogen induced transcription, and the cyclins Ccnl1 and Ccnl2 which are transcriptional regulators of pre-mRNA splicing.

Phenotypic information obtained from independent studies on animals need to be integrated in order to reliably compare results across different mice colonies and laboratory set-ups. The sources of phenotypic data used in the present study are metadatabases in which uniformity criteria and manual curation has been imposed on assembled records. The Mouse Tumor Biology Database (MTB) contains both spontaneous and induced tumor information for over 50 inbred strains, 
which is primarily extracted from the literature, from tumor pathology images submitted by investigators, and from routine animal health screenings of mouse colonies at Jackson Laboratory [9]. Then, is curated with the help of natural language processing tools to cope with increasing amounts of phenotype information in the literature [48]. Similarly, the Mouse Phenome Database (MPD) has developed standards for deposition of phenotypic data of mice including strain purity, study design, animal age and statistical power. Contributors are requested to provide complete measurement descriptions, experimental protocols as well as housing, diet and health status of animals. MPD curates data and computes summary statistics for each measurement in all strains [8].

Results presented here were obtained with a linear regression analysis. However, interplaying gene networks linked to phenotypes may not necessarily follow linear relationships with regard to transcript levels. Recently, a few reports have attempted to identify non-monotone or non-linear phenotype-transcriptome associations. Lin et al. (2008) proposed the coefficient of multiple determination $\left(R^{2}\right)$ of a natural cubic spline regression model [49]. In a related work, three correlation methods (Pearson, Spearman and Hoeffding's D) were compared to analyze co-expressed genes. Hoeffding's D dependence measure was found to be the best suited to identify nonlinear and non-monotonic associations [50]. These types of analytical approaches are needed to uncover causal phenotype-transcriptome connections that do not follow obvious linear behaviors.

Finally, since one of the strains showed a much higher OTF than the other three, we were interested in search for stronger gene links with this phenotype in the SWR strain. A t-test conducted between SWR versus the remaining 3 strains resulted in 530 statistically significant clones (see Additional file 1, figure 1). Of these, 373 clones were common with the regression test while 280 were coincident with the ANOVA test. The overlap between the 3 tests resulted in a list of 266 clones having a functional profile that resembled the terms described in Additional file 1, Table 2 for the regression results. On the other hand, 143 clones were exclusive in the t-test. Reduction of the latter subset resulted in 92 unique gene identities which were subjected to a GO analysis summarized in Additional file 1, table 3. The combined 10-terms list suggests the involvement of intracellular vesicle traffic, protein sorting and actin cytoskeleton dynamics in the observed high OTF of SWR strain. Indeed, oocyte meiotic maturation involves events related to spindle assembly. In somatic cells, chromosome segregation errors during mitosis may contribute to cancer development and progression [51]. The genes App, Aplp2 and Appbp2, all related to the amyloid beta precursor protein, were recurrent in Additional file 1 , table 3. The well known involvement of amyloid beta protein in Alzheimer's disease pathogenesis may actually be due to a chromosomal instability process [52]. Analogous mechanisms may partly explain the high OTF observed in SWR mice.

\section{Conclusion}

This work describes statistically significant variation in ovarian gene expression of four commonly studied mouse strains. We found that over $60 \%$ of these differences are linked to the biological variability observed in spontaneous ovarian tumor rates and reproductive parameters across strains. If NL is equivalent to multiparity, the inverse relationship detected between genes correlated to OTF and NL points to a protective effect of successive pregnancies. Post-transcriptional control of ovarian angiogenesis, folliculogenesis and oocyte maturation seems to be major contributors to this effect. Conversely, overexpression of protein folding genes might be considered as a susceptibility factor. These findings, in addition to the lack of association between OTF and LS -a measure of multiple ovulation- support repetitive menstrual cycling instead of repetitive ovulations as an important contributor to ovarian tumorigenesis. Further experimental research as well as development of bioinformatic and statistical tools to uncover complex phenotype-transcriptome associations is needed.

\section{Methods}

\section{Animals, RNA extraction and cDNA labeling}

Mouse strains BALB/c, C57BL/6, FVB and SWR were maintained at the Laboratory Animal Sciences Program, SAIC-NCI Frederick (Frederick, MD), under protocols of the Institutional Animal Care and Use Committee (IACUC). Adult (8-weeks old) females grown from trio mating-established colonies were euthanised on late metestrus phase by cervical dislocation after gaseous $\mathrm{CO}_{2}$ administration. Whole ovaries from 4-5 animals were removed from surrounding adipose tissue, thereafter pooled and immediately frozen in liquid nitrogen. Total RNA was extracted with Trizol (Invitrogen, CA) and directly labeled as Cyanine- 3 or Cyanine- 5 fluorescent cDNA using reverse transcription under conditions previously described [11].

\section{Microarray experiments}

NIA-15K mouse cDNA microarrays were used. This is a curated collection consisting of 15,261 clones derived from expression libraries obtained from pre- and periimplantation embryos, E12.5 female gonad/mesonephros and newborn ovaries [53]. Microarrays were spotted at the Laboratory of Molecular Technology, SAIC-NCI 
Frederick (Frederick, MD) with a BioRobotics Microgrid arrayer (Genomic Solutions, MI). Hybridization conditions and washes have been described elsewhere [11]. Samples were co-hybridized against a whole-newborn mouse total RNA as common reference sample using a replicated dye-swap design. A total of 24 microarray hybridizations were performed. TIFF images were captured with a GenePix 4000B fluorescent scanner (Molecular Devices, CA) and then saved for further analysis.

\section{Statistical and bioinformatic analysis}

Scanned microarray images were extracted as GPR files using the GenePix 5.0 software and uploaded to the NCI's Microarray Database ("mAdb"; http://nciarray.nci. nih.gov). Data files containing updated gene annotation were subjected to local ("print-tip") loess normalization, scale adjustment, and filtering/imputation of missing values with the "DNMAD" and "PreProcessor" tools at the GEPAS server (http://www.gepas.org). Reproductive and spontaneous ovarian tumor records were extracted from the MPD at http://www.jax.org/phenome[8] and from the MTB at http://tumor.informatics.jax.org/ mtbwi/index.do[9] databases, respectively. Tumor data corresponds to the "highest reported tumor frequency" in all literature records collected in MTB for each strain. Continuous tumor and reproductive data for the 4 mouse strains (see Table 1) were correlated to gene expression $\log _{2}$ ratios using linear regression analysis under multiple-test control (FDR indep) with the tool Pomelo II using 200,000 permutations (http://pomelo2. bioinfo.cnio.es/). The ANOVA test among the 4 strains and a t-test between SWR and the 3 remaining strains were also conducted with Pomelo II using 200,000 permutations. Gene functionality was primarily assessed with Gene Ontology (GO) terms using hypergeometric tests conducted with WebGestalt (http://bioinfo.vanderbilt.edu/webgestalt). Literature mining with HUGO approved gene symbols, associated aliases and keywords, was carried out in PubMed queried through GeneCards (http://www.genecards.org) and with SciMiner (http:// jdrf.neurology.med.umich.edu/SciMiner/).

\section{Quantitative PCR confirmation of microarray results}

Primer pairs design, cDNA preparation, thermocycling conditions and equipment has been previously described [11]. Quantification of mRNAs was based on $C_{T}$ values, which is defined as the PCR cycle at which an increase in reporter fluorescence above baseline signal can be detected. Normalization was done with the $18 \mathrm{~S}$ rRNA as reference transcript assayed under identical conditions respective to the gene of interest in both the test and the reference RNA samples. The $\Delta \Delta \mathrm{C}_{\mathrm{T} \text {-Sample value }}$ $\left(\Delta \Delta \mathrm{C}_{\mathrm{T}-\text { Sample }}=\Delta \Delta \mathrm{C}_{\mathrm{T} \text {-Sample }}-\Delta \mathrm{C}_{\mathrm{T}-\text { Reference }}\right)$ was transformed by taking the result of the expression: If $2^{(-\Delta \Delta C T)}$
$-1>0$ then the result $=2^{(-\Delta \Delta \mathrm{CT})}-1$ or else the result $=$ $-1 / 2^{(-\Delta \Delta C T)}$ This calculation converted the linear range for down regulation from $1 \rightarrow 0$ to $0 \rightarrow(-\infty)$, and up regulation from $1 \rightarrow(+\infty)$ to $0 \rightarrow(+\infty)$ in the $\log _{2}$ scale.

Additional file 1: Contains: Table 1: Detailed microarray ratios and Q-PCR results of selected transcripts. Table 2: Functional analysis of genes correlated to all phenotypes. Table 3: Gene Ontology profile of genes differentially expressed between SWR and the remaining three strains. Figure 1: Summary of statistical tests among ovarian transcriptional profiles of four mouse strains.

Additional file 2: Contains the complete, loess normalized dataset for 14,586 transcripts.

Additional file 3: Contains results for all 145 clones correlated both to OTF and NL in mouse ovaries.

Additional file 4: Functional profile of transcripts correlated both to OTF and NL in the mouse ovary

\section{Acknowledgements}

UU was a fellow at the ORFD Program, OIA, National Cancer Institute (NCI) at National Institutes of Health $(\mathrm{NIH})$. This work was funded in part with Federal Funds from the NCl, NIH under contract N01-C0-12400. The bioinformatic section of this paper was supported by the grant 1205/09-2 from Vicerrectoria de Investigacion y Desarrollo (VID), Universidad de Chile. The pilot Microarray facility at ICBM, Facultad de Medicina, Universidad de Chile has been supported by the grant Mecesup UCH0115. Travel funding to attend the 2009 X-Meeting was granted by VID, Universidad de Chile. This article has been published as part of BMC Genomics Volume 11 Supplement 5, 2010: Proceedings of the 5th International Conference of the Brazilian Association for Bioinformatics and Computational Biology. The full contents of the supplement are available online at http://www. biomedcentral.com/1471-2164/11?issue=S5.

\section{Author details}

'Laboratorio de Genómica Aplicada, ICBM, Universidad de Chile,

Independencia 1027, Santiago, Chile. 'Laboratory of Molecular Technology, SAIC-NCI Frederick, 915 Tollhouse Avenue, Suite 211, Frederick, MD 21701 ${ }^{3}$ Laboratory Animal Sciences Program, SAIC-NCI Frederick, Frederick, MD 21702. ${ }^{4}$ Center for Comparative Medicine, Baylor College of Medicine, One Baylor Plaza, Houston, TX 77030.

\section{Authors' contributions}

UU designed the study, performed microarray experiments, data analysis, interpretation and wrote the manuscript; GAO handled clone collection and manufactured microarrays; GMZ carried out animal care and tissue harvest; JMC performed Q-PCR assays; JJS and DJM participated in study design and coordination, and critically revised the manuscript. All authors read and approved the final manuscript.

\section{Competing interests}

The authors declare that they have no competing interests.

\section{Published: 22 December 2010}

\section{References}

1. Riman T, Nilsson S, Persson IR: Review of epidemiological evidence for reproductive and hormonal factors in relation to the risk of epithelial ovarian malignancies. Acta Obstet Gynecol Scand 2004, 83(9):783-95.

2. Purdie DM, Bain CJ, Siskind V, Webb PM, Green AC: Ovulation and risk of epithelial ovarian cancer. Int J Cancer 2003, 104:228-32.

3. Choi JH, Wong AS, Huang HF, Leung PC: Gonadotropins and ovarian cancer. Endocr Rev 2007, 28(4):440-61.

4. Garson K, Shaw TJ, Clark KV, Yao DS, Vanderhyden BC: Models of ovarian cancer-are we there yet? Mol Cell Endocrinol 2005, 239(1-2):15-26.

5. Silver LM: Mouse genetics. Concepts and applications. Oxford University Press; 1995. 
6. VomSaal FS, Finch CE, Nelson JF: Natural history and mechanism of reproductive ageing in humans, laboratory rodents and other selected vertebrates. In The Physiology of Reproduction.. 2 edition. Raven Press, New York;Knobil and Neilleds 1994:

7. Clow OL, Hurst PR, Fleming JS: Changes in the mouse ovarian surface epithelium with age and ovulation number. Mol Cell Endocrinol 2002, 191(1):105-11.

8. Bogue MA, Grubb SC, Maddatu TP, Bult CJ: Mouse Phenome Database (MPD). Nucleic Acids Res 2007, 35(Database issue):D643-9.

9. Begley DA, Krupke DM, Vincent MJ, Sundberg JP, Bult CJ, Eppig JT: Mouse Tumor Biology Database (MTB): status update and future directions. Nucleic Acids Res 2007, , Database issue: D638-42.

10. Liang $Y$, Kelemen A: Associating phenotypes with molecular events: recent statistical advances and challenges underpinning microarray experiments. Funct Integr Genomics 2006, 6(1):1-13.

11. Urzua U, Roby KF, Gangi LM, Cherry JM, Powell II, Munroe DJ: Transcriptomic analysis of an in vitro murine model of ovarian carcinoma: functional similarity to the human disease and identification of prospective tumoral markers and targets. J Cell Physiol 2006, 206(3):594-602.

12. Urzua U, Frankenberger C, Gangi L, Mayer S, Burkett S, Munroe DJ: Microarray comparative genomic hybridization profile of a murine model for epithelial ovarian cancer reveals genomic imbalances resembling human ovarian carcinomas. Tumour Biol 2005, 26(5):236-44

13. Burns KH, Owens GE, Ogbonna SC, Nilson JH, Matzuk MM: Expression profiling analyses of gonadotropin responses and tumor development in the absence of inhibins. Endocrinology 2003, 144(10):4492-507.

14. Stylianou IM, Clinton M, Keightley PD, Pritchard C, Tymowska-Lalanne Z, Bünger L, Horvat S: Microarray gene expression analysis of the Fob3b obesity QTL identifies positional candidate gene Sqle and perturbed cholesterol and glycolysis pathways. Physiol Genomics 2005, 20(3):224-32

15. Hunter SM, Mansergh FC, Evans MJ: Optimization of minuscule samples for use with cDNA microarrays. J Biochem Biophys Methods 2008, 70(6):1048-58.

16. Yang YH, Dudoit S, Luu P, Lin DM, Peng V, Ngai J, Speed TP: Normalization for CDNA microarray data: a robust composite method addressing single and multiple slide systematic variation. Nucleic Acids Res 2002, 30(4):e15.

17. Wang Y, Barbacioru C, Hyland F, Xiao W, Hunkapiller KL, Blake J, Chan F, Gonzalez C, Zhang L, Samaha RR: Large scale real-time PCR validation on gene expression measurements from two commercial longoligonucleotide microarrays. BMC Genomics 2006, 7:59.

18. Okazaki N, Kikuno R, Ohara R, Inamoto S, Koseki H, Hiraoka S, Saga Y, Nagase T, Ohara O, Koga $\mathrm{H}$ : Prediction of the coding sequences of mouse homologues of KIAA gene: III. The complete nucleotide sequences of 500 mouse KIAA-homologous cDNAs identified by screening of terminal sequences of cDNA clones randomly sampled from size-fractionated libraries. DNA Res 2003, 10(4):167-80.

19. Macario AJ, Conway de Macario E: Molecular chaperones: multiple functions, pathologies, and potential applications. Front Biosci 2007, 12:2588-600.

20. Manna PR, Dyson MT, Stocco DM: Regulation of the steroidogenic acute regulatory protein gene expression: present and future perspectives. $\mathrm{Mol}$ Hum Reprod 2009, 15(6):321-33.

21. Sohn KC, Lee KY, Park JE, Do SI: OGT functions as a catalytic chaperone under heat stress response: a unique defense role of OGT in hyperthermia. Biochem Biophys Res Commun 2004, 322(3):1045-51.

22. Fielding P, Turnbull L, Prime W, Walshaw M, Field JK: Heterogeneous nuclear ribonucleoprotein A2/B1 up-regulation in bronchial lavage specimens: a clinical marker of early lung cancer detection. Clin Cancer Res 1999, 5:4048-52.

23. Huang CS, Shen CY, Wang HW, Wu PE, Cheng CW: Increased expression of SRp40 affecting CD44 splicing is associated with the clinical outcome of lymph node metastasis in human breast cancer. Clin Chim Acta 2007, 384(1-2):69-74.

24. Saha S, Murthy S, Rangarajan PN: Identification and characterization of a virus-inducible non-coding RNA in mouse brain. J Gen Virol 2006, 87(Pt 7):1991-5.

25. Perez DS, Hoage TR, Pritchett JR, Ducharme-Smith AL, Halling ML, Ganapathiraju SC, Streng PS, Smith DI: Long, abundantly expressed noncoding transcripts are altered in cancer. Hum Mol Genet 2008, $17(5): 642-55$
26. Velazquez-Dones A, Hagopian JC, Ma CT, Zhong XY, Zhou H, Ghosh G, Fu XD, Adams JA: Mass spectrometric and kinetic analysis of ASF/SF2 phosphorylation by SRPK1 and Clk/Sty. J Biol Chem 2005, 280(50):41761-8.

27. Kayampilly PP, Menon KM: Follicle-stimulating hormone increases tuberin phosphorylation and mammalian target of rapamycin signaling through an extracellular signal-regulated kinase-dependent pathway in rat granulosa cells. Endocrinology 2007, 148(8):3950-7.

28. Fan HY, Sun QY: Involvement of mitogen-activated protein kinase cascade during oocyte maturation and fertilization in mammals. Biol Reprod 2004, 70(3):535-47.

29. Fan HY, Shimada M, Liu Z, Cahill N, Noma N, Wu Y, Gossen J, Richards JS: Selective expression of KrasG12D in granulosa cells of the mouse ovary causes defects in follicle development and ovulation. Development 2008 135(12):2127-37

30. Gallardo TD, John GB, Shirley L, Contreras CM, Akbay EA, Haynie JM Ward SE, Shidler MJ, Castrillon DH: Genomewide discovery and classification of candidate ovarian fertility genes in the mouse. Genetics 2007, 177(1):179-94.

31. Zhang H, Xu B, Xie H, Zhou B, Ouyang H, Ning G, Li G, Zhang M, Xia G: Lanosterol metabolic product(s) is involved in primordial folliculogenesis and establishment of primordial follicle pool in mouse fetal ovary. $\mathrm{Mol}$ Reprod Dev 2009, 76(5):514-21.

32. Brown MS, Goldstein JL: A proteolytic pathway that controls the cholesterol content of membranes, cells, and blood. Proc Natl Acad Sci U S A 1999, 96(20):11041-8

33. Durcova-Hills G, Capel B: Development of germ cells in the mouse. Curr Top Dev Biol 2008, 83:185-212.

34. Meidan R, Levy N: Endothelin-1 receptors and biosynthesis in the corpus luteum: molecular and physiological implications. Domest Anim Endocrinol 2002, 23(1-2):287-98.

35. Bamberger ES, Perrett CW: Angiogenesis in epithelian ovarian cancer. Mol Pathol 2002, 55(6):348-59.

36. Gordan JD, Simon MC: Hypoxia-inducible factors: central regulators of the tumor phenotype. Curr Opin Genet Dev 2007, 17(1):71-7.

37. Pocar P, Fischer B, Klonisch T, Hombach-Klonisch S: Molecular interactions of the aryl hydrocarbon receptor and its biological and toxicological relevance for reproduction. Reproduction 2005, 129(4):379-89.

38. Jinnin M, Medici D, Park L, Limaye $N$, Liu Y, Boscolo E, Bischoff J, Vikkula M, Boye E, Olsen BR: Suppressed NFAT-dependent VEGFR1 expression and constitutive VEGFR2 signaling in infantile hemangioma. Nat Med 2008, 14(11):1236-46.

39. Nowak DG, Woolard J, Amin EM, Konopatskaya O, Saleem MA, Churchill AJ, Ladomery MR, Harper SJ, Bates DO: Expression of pro- and antiangiogenic isoforms of VEGF is differentially regulated by splicing and growth factors. J Cell Sci 2008, 121(Pt 20):3487-95

40. Dowhan DH, Hong EP, Auboeuf D, Dennis AP, Wilson MM, Berget SM, O'Malley B.W: Steroid hormone receptor coactivation and alternative RNA splicing by U2AF65-related proteins CAPERalpha and CAPERbeta. Mol Cell 2005, 17(3):429-39.

41. Jung DJ, Na SY, Na DS, Lee JW: Molecular cloning and characterization of CAPER, a novel coactivator of activating protein- 1 and estrogen receptors. J Biol Chem 2002, 277(2):1229-34.

42. Fuller-Pace FV, Ali S: The DEAD box RNA helicases p68 (Ddx5) and p72 (Ddx17): novel transcriptional co-regulators. Biochem Soc Trans 2008 36(Pt 4):609-12.

43. Cobreros L, Fernández-Miñán A, Luque CM, González-Reyes A, MartínBermudo MD: A role for the chaperone $\mathrm{Hsp70}$ in the regulation of border cell migration in the Drosophila ovary. Mech Dev 2008, 125(1112):1048-58.

44. Matsuzaki S, Canis M, Pouly JL, Botchorishvili R, Déchelotte PJ, Mage G: Differential expression of genes in eutopic and ectopic endometrium from patients with ovarian endometriosis. Fertil Steril 2006, 86(3):548-53.

45. Saha SK, Ghosh P, Bhattacharya S, Roy SS: Procollagen synthesis is increased in hypothyroid rat ovary by a parallel and compensatory pathway. Cell Physiol Biochem 2007, 19(5-6):313-22.

46. Racki WJ, Richter JD: CPEB controls oocyte growth and follicle development in the mouse. Development 2006, 133(22):4527-37.

47. Perlman S, Bouquin T, van den Hazel B, Jensen TH, Schambye HT, Knudsen S, Okkels JS: Transcriptome analysis of FSH and FSH variant stimulation in granulosa cells from IVM patients reveals novel regulated genes. Mol Hum Reprod 2006, 12(3):135-44. 
48. Xu H, Krupke D, Blake J, Friedman C: A natural language processing (NLP) tool to assist in the curation of the laboratory Mouse Tumor Biology Database. AMIA Annu Symp Proc 2006, 1150.

49. Lin R, Dai S, Irwin RD, Heinloth AN, Boorman GA, Li L: Gene set enrichment analysis for non-monotone association and multiple experimental categories. BMC Bioinformatics 2008, 9:481

50. Fujita A, Sato JR, Demasi MA, Sogayar MC, Ferreira CE, Miyano S: Comparing Pearson, Spearman and Hoeffding's D measure for gene expression association analysis. J Bioinform Comput Biol 2009, 7(4):663-84

51. Wang WH, Sun QY: Meiotic spindle, spindle checkpoint and embryonic aneuploidy. Front Biosci 2006, 11:620-36.

52. Thomas P, Fenech M: A review of genome mutation and Alzheimer's disease. Mutagenesis 2007, 22(1):15-33, Epub 2006 Dec 8.

53. Tanaka TS, Jaradat SA, Lim MK, Kargul GJ, Wang X, Grahovac MJ, Pantano S, Sano Y, Piao Y, Nagaraja R, Doi H, Wood WH 3rd, Becker KG, Ko MS: Genome-wide expression profiling of mid-gestation placenta and embryo using a 15,000 mouse developmental CDNA microarray. Proc Natl Acad Sci U S A 2000, 97(16):9127-32.

doi:10.1186/1471-2164-11-S5-S1

Cite this article as: Urzúa et al.: Tumor and reproductive traits are linked by RNA metabolism genes in the mouse ovary: a transcriptome-

phenotype association analysis. BMC Genomics 2010 11(Suppl 5):S1

\section{Submit your next manuscript to BioMed Central} and take full advantage of:

- Convenient online submission

- Thorough peer review

- No space constraints or color figure charges

- Immediate publication on acceptance

- Inclusion in PubMed, CAS, Scopus and Google Scholar

- Research which is freely available for redistribution

Submit your manuscript at www.biomedcentral.com/submit
C Biomed Central 